Le livre, emblème de l'exposition littéraire? Enjeux croisés de la muséalisation ${ }^{\mathrm{I}}$ du livre et de la littérature

\title{
Marie-Clémence Régnier*
}

Dans un numéro récent de la revue Littérature, paru en 20I0, Olivia Rosenthal et Lionel Ruffel s'intéressaient "à ces pratiques littéraires multiples (performances, lectures publiques, interventions sur le territoire, travaux sonores ou visuels) [...] déjou[ant] le mode de reconnaissance de la littérature par l'imprimé et par le livre ${ }^{2} »$. Le titre du numéro? «La littérature exposée».

A contrario, mon analyse prend au mot le titre choisi par la revue et aborde la mise en exposition de la littérature en contexte muséal pour interroger la place qu'y occupe le livre précisément. L'exposition de la littérature n'a, de fait, rien d'évident en soi. Considérée comme un domaine de la pensée avant tout intellectuel et immatériel, la littérature est censée se lire, se discuter, s'interpréter. Son support habituel depuis des siècles, le livre papier, s'expose. Mais tout texte écrit est alors susceptible d'être exposé dans ce cas. De même, le manuscrit pourrait tout aussi bien être considéré comme le support premier de la littérature, au moins sur le plan génétique. Le but de cet article est donc double: il vise à poser les premiers jalons d'une réflexion portant sur les modalités d'exposition de la littérature, d'un point de vue théorique et pratique. Par ailleurs, il tend à dégager quelques «tendances» fortes de l'exposition de l'imprimé en confrontant les origines du phénomène à des réalisations récentes. L'enjeu principal de l'analyse réside ainsi dans les deux questions suivantes : comment

Le terme désigne «l'opération tendant à extraire, physiquement et conceptuellement, une chose de son milieu naturel ou culturel d'origine et à lui donner un statut muséal, la transformer en musealium ou muséalie, objet de musée, soit à la faire entrer dans le champ du muséal ». Voir François Mairesse, "Muséalisation", dans André Desvallées et François Mairesse (dir.), Dictionnaire encyclopédique de muséologie, Paris, Armand Colin, 20II, p. 25I.

* Ancienne élève de l'École normale supérieure de Paris, Marie-Clémence Régnier est agrégée de lettres modernes et diplômée de l'Institut des sciences politiques de Paris. Elle réalise actuellement une thèse à la Sorbonne sur les maisons d'écrivains et la muséalisation de la littérature en France (I797-1937) sous la direction de Florence Naugrette et de Françoise Mélonio.

2 Olivia Rosenthal et Lionel Ruffel, "Introduction», Littérature, vol. 4, n ${ }^{\circ}$ I60, 2OIO, p. 4. 
exposer la littérature? Pourquoi le livre continue-t-il à occuper une place aussi importante dans les expositions littéraires, alors même que d'autres objets s'avèrent plus "spectaculaires ${ }^{3}$ " que lui? L'hypothèse de travail développée tend vers ces deux éléments de réponse: le livre rend la littérature exposable d'un point de vue tangible d'une part; ensuite, la «culture livre» est au cœur de ce qui constitue au plan sensible, symbolique et pour ainsi dire intellectuel le concept et l'expérience de la «littérature» dans notre société d'autre part.

De la sorte, l'étude entend aborder les expositions littéraires par d'autres voies que celles qui ont été privilégiées jusque-là : nomenclature d'expositions organisées par telle institution ${ }^{4}$, évocation descriptive d'expositions dans des dossiers ou des articles de presse réflexions portant sur des problématiques de conservation et de valorisation de collections ${ }^{6}$ ou encore travail portant sur le cas singulier des manuscrits littéraires ${ }^{7}$. Enfin, si un fil historique fournira une trame à l'ensemble, il ne sera toutefois pas question ici de se livrer à un travail historiographique, pas plus que de se livrer à un recensement exhaustif de toutes les expositions littéraires dans le monde. Les bornes chronologiques établies permettront de mettre en avant les principales lignes de force de l'exposition de la littérature. La dimension panoramique de l'analyse permettra ainsi de mettre en perspective l'affirmation du musée moderne révolutionnaire et du culte rendu au livre tout au long du $\mathrm{XIX}^{\mathrm{e}}$ siècle avec les récentes évolutions en

3 Voir François Mairesse, Le musée, temple spectaculaire. Une histoire du projet muséal, Lyon, Presses universitaires de Lyon, coll. "Muséologies», 2002, 215 p.

4 Voir notamment Judith Meyer-Petit, "La maison de Balzac et les paradoxes du musée littéraire", Revue d'histoire littéraire de la France, vol. 95, n' I, I995, p. 59-68 et "Muséographie d'un musée littéraire : la maison de Balzac», Bulletin d'informations de l'ABF, $\mathrm{n}^{\circ} \mathrm{173}, \mathrm{1996}$, p. I012.

5 La Bibliothèque nationale de France $(\mathrm{BnF})$ s'est dotée récemment d'un précieux outil d'inventaire de ses expositions, disponible en ligne sur son site internet.

6 Roland Schaer, "La bibliothèque, lieu d'exposition?", dans Viviane Cabannes et Martine Poulain (dir.), L'action culturelle en bibliothèque, Paris, Éditions du Cercle de la Librairie, I998, p. 23-28; Martine Blanc-Montmayeur, Viviane Cabannes, Jean-Louis Déotte, Jean Hébrard et al., Le musée et la bibliothèque, vrais parents ou faux amis, Paris, Bibliothèque publique d'information, I997, 243 p.; Journées d'étude des 28 mars 2008 et 27 mars 2009 organisées par la Fédération des Maisons d'écrivains et des patrimoines littéraires, respectivement intitulées Les fonds littéraires et Archives d'écrivains: conservation et valorisation des collections, www.litterature-lieux.com.

7 Thomas Cazentre, "Exposer le manuscrit littéraire», mémoire d'étude, Villeurbanne, École nationale supérieure des sciences de l'information et des bibliothèques, 2008, $87 \mathrm{p}$. 
termes de numérisation et de "dématérialisation». Afin de respecter une certaine cohérence nationale, on s'interrogera de fait sur quelques cas délimités et majoritairement français. De fait, la France est un cas d'étude intéressant dans la mesure où elle intègre progressivement à une position traditionnelle sur le rôle du livre dans la valorisation muséale du littéraire une dynamique numérique et multimédia. En outre, son histoire l'associe intimement aux origines du concept et des premières réalisations muséales à la Renaissance puis sous la Révolution française; l'emprise des modalités de constitution de son histoire patrimoniale contraint peut-être à bien des égards sa position à l'égard des nouvelles pratiques liées au livre. Le rapprochement final opéré avec un exemple québécois permettra d'établir un dialogue avec un modèle à la fois proche et distinct de la culture française : la construction d'une identité québécoise singulière bénéficie en effet des expérimentations inédites menées dans le domaine de l'exposition muséale et d'un rapport au patrimoine différent.

Ainsi, après un premier temps théorique consacré aux enjeux fondateurs de l'exposition du livre en général, un second temps traitera la question dans le contexte littéraire. Enfin, un dernier moment présentera la sortie hors du livre ${ }^{8}$ au prisme de plusieurs expositions contemporaines et s'interrogera sur la portée de ce phénomène.

\section{Le culte du livre et de l'écrivain, prélude à la muséalisation de la littérature}

L'exposition du livre repose sur trois présupposés : l'adéquation entre un contenu (littéraire) et un support (le livre), le sacre de l'écrivain ${ }^{9}$ et la valeur patrimoniale du fonds ou de la collection valorisé(e).

\section{Origines de l'exposition du livre}

Le terme de «monstration ${ }^{\mathrm{IO}}$ " s'avère sans doute être le plus pertinent pour désigner la démarche édifiante des collectionneurs et des

8 David Ruffel, "Une littérature contextuelle», Littérature, vol. 4, nº I60, 20I0, p. 6I-73.

9 Voir Paul Bénichou, Le sacre de l'écrivain: essai sur l'avènement d'un pouvoir spirituel lä̈que dans la France moderne, Paris, José Corti, 1973, 492 p.

Io Ce terme est celui qu'emploient les conservateurs du Département des manuscrits de la Bibliothèque nationale de France pour désigner les premières expériences 
institutions patrimoniales qui visent à montrer des collections à un public à la fin du $\mathrm{XIx}^{\mathrm{e}}$ siècle, avant l'affirmation de dispositifs muséographiques plus élaborés au tournant des années 1920-30 ${ }^{\mathrm{II}}$. Elle consiste essentiellement à montrer un trésor de bibliothèque dans une vitrine-écrin, ou une sélection homogène d'imprimés et de manuscrits dans des vitrines à l'occasion d'un événement commémoratif par exemple. L'ensemble s'articule autour de la description des pièces présentées, d'un thème ou de la biographie de l'auteur, au fil de laquelle sont mentionnées les œuvres qui définissent le canon de l'époque. Ce phénomène n'a pourtant rien de nouveau. De fait, les livres occupaient déjà une place de choix ${ }^{\mathrm{I2}}$ dans les premiers cabinets de curiosités, à l'origine des musées tels qu'on les conçoit. Toutefois, la dimension sacrée du culte aux reliques, aux objets du passé et du savoir, qui connaît un net regain au XIX ${ }^{\mathrm{e}}$ siècle $^{\mathrm{I3}}$, tend à redonner à ces pratiques un nouvel essor.

\section{Le livre-monument}

La monstration se fonde sur la pratique individuelle, dynastique ou curiale de la collection à la Renaissance dans les cabinets de curiosités ${ }^{\mathrm{I4}}$ et dans les bibliothèques ${ }^{\mathrm{IS}}$. La monumentalisation symbolique du livre et la présentation magistrale de l'objet sous vitrine par le passé constituent les fondements de la muséalisation du livre à l'époque

d'expositions de l'établissement au XIx $\mathrm{X}^{\mathrm{e}}$ siècle, ainsi que je l'ai appris lors d'un stage effectué en 2007 dans ce service. Sur l'inventaire de la BnF (voir note 5) figure également le mot "présentation", peut-être moins évocateur.

II François Poncelet, "Regards actuels sur la muséographie d'entre-deux-guerres", CeROArt, vol. 2, 4 octobre 2008, http://ceroart.revues.org/565.

I2 Sur la question, deux ouvrages essentiels: le traité de Samuel Quiccheberg, Inscriptiones vel Tituli theatri Amplissimi, Munich, ex officina Adami Berg typographi, 1565, qui fixe les contours de la collection encyclopédique à la Renaissance; et la synthèse récente de Dominique Moncond'huy et al., La licorne et le bézoard: une histoire des cabinets de curiosités: exposition, Poitiers, Musée Sainte-Croix, et Espace Mendès-France, I8 octobre 2013 - I6 mars 20I4, cat. exp., Montreuil, Gourcuff Gradenigo, 2013, 5II p.

I3 Voir Jean-Claude Bonnet, Naissance du Panthéon. Essai sur le culte des grands hommes, Paris, Fayard, coll. "L'esprit de la cité», I998, 4I4 p.; Bénichou, Le sacre de l'écrivain.

${ }^{14}$ Krzysztof Pomian, Collectionneurs, amateurs et curieux. Paris, Venise: XVI ${ }^{e}-$ XVIII ${ }^{e}$ siècle, Paris, Gallimard, 1987, 368 p.

Is Voir notamment l'Histoire des bibliothèques françaises, Paris, Éditions du Cercle de la Librairie, I988-92, 4 t. 
moderne. Mais la monstration acquiert une dimension publique avec la patrimonialisation de l'auteur et de l'écrit à partir du XVIII ${ }^{\mathrm{e}}$ siècle et plus particulièrement de la Révolution française ${ }^{\mathrm{I} 6}$. Le caractère tangible et symbolique du livre, sa relative résistance matérielle ainsi que la lisibilité des caractères imprimés par rapport à d'autres supports ${ }^{17}$ expliquent sa présence prédominante au sein des espaces d'exposition. Le livre concentre en outre une valeur patrimoniale protéiforme : une valeur testimoniale et historique, une valeur «technique» et artisanale résidant dans un certain savoir-faire, une valeur esthétique (dont le livre $\mathrm{d}^{\prime} \operatorname{art}^{\mathrm{I} 8}$ constitue un aboutissement), une valeur sentimentale (des annotations manuscrites, des traces d'appropriation du livre conferent au livre une dimension émotionnelle forte). Enfin, une valeur cultuelle se surimpose à ces éléments (le livre peut être considéré comme la relique d'un individu célèbre, lecteur ou collectionneur de renom).

La notion d' "aura», développée par Walter Benjamin ${ }^{19}$, paraît pouvoir résumer dans un premier temps l'intérêt muséal qui entoure le livre. En ce sens, le hic et nunc de l'œuvre d'art ne s'applique pas au livre dans les mêmes termes. Si les livres imprimés avant l'ère de l'industrialisation de l'édition constituent des exemplaires "uniques" ayant échappé à la perte ou à la destruction, le livre semble être depuis lors le bien culturel reproductible par excellence. Par conséquent, le livre a intrinsèquement une "valeur d'exposition" élevée, au sens où Benjamin emploie l'expression pour désigner la valeur commerciale de l'objet produit en série et pris dans le jeu de l'industrialisation. Au titre des autres valeurs évoquées ci-dessus, le livre entre donc en musée lorsqu'il perd son usage de support du texte et de l'œuvre et acquiert une nouvelle fonction. On rejoint alors la définition de l'objet

i6 Marie-Clémence Régnier, "Maisons d'écrivains et expositions littéraires: le Musée de la littérature aujourd'hui», mémoire de master I, Paris, Université Paris-Sorbonne, 2008, 95 p.

17 Voir l'exposition Brouillons d'écrivains en 2010 à la $\mathrm{BnF}$ constituée essentiellement de manuscrits; voir Thomas Cazentre, "Exposer le manuscrit littéraire».

I8 Nom désignant les livres où les illustrations de nature artistique occupent une place prépondérante par rapport au texte, ou dans lequel ces deux composantes sont harmonieusement disposées sur la page, avec d'éventuels effets de papier spectaculaires. Depuis la seconde moitié du $\mathrm{XIX}^{\mathrm{e}}$ siècle, le livre d'art joue un grand rôle dans l'affirmation des arts décoratifs et de la pratique de l'écriture comme art graphique à mi-chemin entre littérature et beaux-arts. Voir Stéphanie Bernier, Sophie Drouin et Josée Vincent (dir.), Le livre comme art-Matérialité et sens, Québec, Éditions Nota Bene, 2013, 212 p.

I9 Walter Benjamin, "L'œuvre d'art à l'époque de sa reproductibilité technique» [1939], dans Euvres III, Paris, Gallimard, 2000, I344 p. 
muséal de Krzysztof Pomian autour de la notion de "sémiophore ${ }^{20}$ ", qui s'avère ici centrale. Par là, l'historien désigne effectivement un objet sans utilité et maintenu à l'écart des activités économiques, situation d'où il tire précisément sa valeur.

Par conséquent, plus la "valeur d'exposition" du livre est élevée (moins le livre est rare), plus sa valeur cultuelle et sa légitimité à entrer au musée sont potentiellement faibles. De la sorte, l'aura du livre exposé trouve son sens le plus profond dans son degré d'historicité et, plus encore, dans la coïncidence historique entre l'écriture du texte d'une part et la fabrication-parution du livre d'autre part. Mieux, plus le livre est ancien et rare, plus son exposition est donc intéressante et légitime : rien de mieux, dans le cas qui nous occupe, qu'un livre paru du vivant de Jean Racine par exemple (et cas idéal, annoté de sa main) pour alimenter une exposition portant sur le dramaturge classique. En fin de compte, l'aura d'un livre exposé résiderait dans le fait qu'il tend à échapper à sa nature reproductible et à gagner une forme d'unicité dans la série et dans la masse des livres.

\section{La sanctuarisation de l'écrivain et du livre imprimé au sein de l'exposition}

À l'heure du plein essor de l'imprimé, du lectorat et des innovations techniques entourant sa reproduction, valorisées lors des Expositions universelles ${ }^{21}$, il semble donc que l'exposition muséale opère une forme de sanctuarisation autour du livre muséalisé. La sacralisation du livre le soustrait en quelque sorte aux conséquences néfastes ${ }^{22}$ imputées à son industrialisation et à sa publicisation à outrance au regard de nombreux contemporains ${ }^{23}$, quand, pour d'autres, l'exposition nourrit le rêve de diffusion de la mythique République des Lettres auprès

\footnotetext{
20 Pomian, Collectionneurs, amateurs et curieux.

2I Pascal Ory, Les Expositions universelles de Paris, Paris, Ramsay, 1982, I57 p.

22 Nathalie Heinich, "L'aura de Walter Benjamin", Actes de la recherche en sciences sociales, vol. 49, 1983, p. I07-I09.

23 En ce sens, Jules Janin dénie de façon provocante au livre bon marché sa nature de "livre" qu'il décrit comme un objet noble, digne du grand auteur et du collectionneur de goût : " un bouquin taché de lie, où la fille errante et le laquais fangeux ont laissé la trace ineffaçable de leurs doigts malpropres [...] : livre! une loque infecte, un haillon qui n'a plus de nom dans aucune langue!». Jules Janin, L'amour des livres, Paris, J. Miard, I866, p. 8-9.
} 
d'un public idéalement élargi et pour lequel le musée constitue le nouveau temple ${ }^{24}$.

L'étroite association entre livres rares, livres à charge symbolique forte («classiques» et «trésors» de bibliothèque), et grands écrivains au sein de l'exposition littéraire paraît, quant à elle, confier au culte du grand écrivain la promotion d'un certain art du livre, et de pratiques d'écriture ou bien de lecture d'excellence ${ }^{25}$. De fait, si l'écrivain n'est pas le seul dépositaire de cette conception, il semble bien tout de même qu'il faille voir dans sa propre aura et dans son rapport intime à l'écrit et à la langue un motif d'élection parmi d'autres grands hommes (artistes, hommes politiques et historiques, savants).

\section{La «culture livre» de la littérature, noyau dur de l'exposition littéraire?}

Le processus d'autonomisation dans lequel s'affirme le champ littéraire tout au long du XIX ${ }^{\mathrm{e}}$ siècle opère un recentrage des divers genres (art dramatique, poésie, épopée et roman) qui composent les Belles-Lettres autour de l'idée de "littérature», incarnée par l'écrivain précisément. À l'heure de la conceptualisation théorique et méthodologique de la discipline littéraire ${ }^{26}$, ancrée par tradition dans l'écrit (rédigé, lu et interprété), la littérature s'affirme ainsi autour de l'«Auteur» sous l'influence déterminante du mouvement romantique.

\section{Naissance de l'exposition littéraire}

L'autorité du "grand écrivain " plus particulièrement, ainsi que le culte qui entoure sa maison (bientôt transformée en musée dans le dernier

24 Voir notamment dans le contexte du Front populaire, Julien Cain (dir.), Ébauches et premiers éléments d'un musée de la littérature, préface de Paul Valéry, Paris, Denoël, 1937, 92 p.

25 La sélection des livres exposés implique par conséquent une hiérarchie non seulement entre des catégories de livres (la "grande littérature» et les livres rares sont davantage valorisés par l'exposition), mais aussi entre les auteurs (les "classiques" et les "grands" auteurs sont placés sous les feux de la rampe) et certainement entre les lecteurs. Une étude comparative des publics des expositions littéraires au XIX ${ }^{\mathrm{e}}$ siècle et des expositions au cours du $\mathrm{Xx}^{\mathrm{e}}$ siècle reste à faire.

26 Antoine Compagnon, "Comment on devient un grand écrivain français", Le Temps de la réflexion, $\mathrm{n}^{\circ}$ 3, 1982, pp. 361-380; et La Troisième République des Lettres, Paris, Éditions du Seuil, I983, 38I p. 
tiers du XIX ${ }^{\mathrm{e}}$ siècle en France), expliquent le culte qui se dessine autour de ses manuscrits, des objets de son quotidien (considérés comme des reliques) et de sa bibliothèque tout au long du XIx ${ }^{\mathrm{e}}$ siècle. C'est dans ce contexte que les maisons d'écrivains et les bibliothèques en France (à commencer par la plus grande d'entre elle, la Bibliothèque nationale dans le dernier tiers du XIX ${ }^{\mathrm{e}}$ siècle là encore ${ }^{27}$ ) ont commencé à mettre à l'honneur le livre de littérature, aux côtés d'autres éléments tirés des collections (manuscrits, estampes, portraits, reliques).

\section{Statut du livre dans l'exposition littéraire}

Si l'exposition littéraire n'a pas le monopole du livre et de sa valorisation, l'identification (en réalité historique) entre œuvre littéraire, écrivain et livre tend toutefois à se naturaliser. Plus précisément, l'exposition littéraire adopte une voie propre à l'histoire littéraire en commémorant les auteurs classiques dans un premier temps puis les auteurs canoniques du XIX ${ }^{\mathrm{e}}$ siècle, entrés au panthéon de la littérature nationale sous l'effet de la médiation des institutions scolaires et universitaires ${ }^{28}$. Le culte de certains auteurs contribue en effet à fixer autour de l'archive et de l'écrit en général une fonction de représentation du génie national depuis la Révolution française ${ }^{29}$. Dans cet ordre des choses, le livre occupe une place centrale puisqu'il figure le rôle déterminant de l'œuvre littéraire dans la diffusion de la gloire de l'écrivain et de la nation. La promotion des collections livresques au sein du musée répond ainsi à trois fonctions principales qu'on attribue au livre littéraire:

- le livre est exposé en ce qu'il représente le trait d'union de la relation auteur/lecteur-visiteur autour de l'œuvre dont il est le support;

27 Sur ce point, il convient de préciser que la bibliothèque donne la part belle au livre, beaucoup plus sans doute que les maisons d'écrivains, dont le statut de maison-musée repose sur la valorisation de divers objets du quotidien et de reliques.

28 Voir notamment Antoine Compagnon, La Troisième République des Lettres; Alain Viala, "Qu'est-ce qu'un classique?», Bulletin des bibliothèques de France, $\mathrm{n}^{\mathrm{o}} \mathrm{I}$, I992, http://bbf.enssib.fr/consulter/bbf-I992-OI-O0o6-oor; Stéphane Zékian, L'invention des classiques. Le siècle de Louis XIV existe-t-il?, Paris, CNRS éditions, 20I2, 384 p.

29 Anne-Marie Thiesse, La Création des Identités nationales. Europe, XIX $-X X^{e}$ siècle. Paris, Seuil, 1999, 302 p. 
- il constitue un élément central d'identification du statut de l'écrivain et de sa renommée;

- il contribue à établir la reconnaissance de l'écrivain au travers des nombreuses traductions et rééditions dont une œuvre fait l'objet.

De ce fait, le livre détient une valeur édifiante; il est le signe de la gloire de l'écrivain au sein de l'espace symbolique, social et économique.

\section{La fin de l'autorité du livre et de l'auteur?}

Les expositions réalisées aujourd'hui s'inscrivent dans la continuité du modèle historique en valorisant toujours les grandes figures de la littérature moderne et les livres-trésors. Toutefois la contestation de l'auctorialité et de la culture livresque depuis la seconde moitié du $\mathrm{xx}^{\mathrm{e}}$ siècle, ainsi que des impératifs d'ordre politique et économique $\mathrm{e}^{30}$ incite les institutions patrimoniales à diversifier et à moderniser les assises de l'exposition littéraire. On note en effet que les expositions accordent beaucoup d'attention à la figure de l'écrivain au détour d'un ou plusieurs thème(s) de société; ce faisant, elles interrogent la réception de l'œuvre et les ressorts du passage à la postéritée ${ }^{3 \mathrm{I}}$. Elles mettent également en lumière des cycles littéraires et des mythes ${ }^{32}$ spectaculaires, ou des auteurs d'avant-garde ou à la marge ${ }^{33}$ de la culture du grand public auquel elles font découvrir des pans méconnus de la littérature. Ces nouvelles orientations introduisent des changements de statut du livre au sein de l'exposition: les grands classiques et les trésors de bibliothèque présentés de façon

30 Depuis les années 1980, l'impératif d'accessibilité à la culture, au savoir et au patrimoine au plus grand nombre a accentué et accéléré la conversion des missions de conservation de la bibliothèque et de la maison-musée en faveur d'une mission de valorisation de la lecture et du livre passant par la réalisation d'exposition notamment.

31 Pour le premier aspect, voir la Maison natale de Victor Hugo à Besançon qui se concentre sur l'engagement politique et social de l'écrivain; pour le second, l'exposition Les misérables, un roman inconnu?, Paris, Maison de Victor Hugo, IO octobre $2008-\mathrm{I}^{\mathrm{er}}$ février 2009; ou Balzac vu d'ailleurs. Un regard taïwanais sur La comédie humaine, Paris, Maison de Balzac, ${ }^{\text {er }}$ mars - 26 mai 2013.

32 Exposition La légende du roi Arthur, Paris, BnF, 20 octobre 2009 - 24 janvier 2oIo par exemple.

33 Tels Antonin Artaud, dessins, Paris, Centre Pompidou, I ${ }^{\text {er }}$ juillet-II octobre 1987; Antonin Artaud, Paris, BnF, 7 novembre 2006 - 4 février 2007; ou Pierre-Jean Jouve, Paris, $\mathrm{BnF}, 24$ septembre - Io novembre 2013. 
monumentale ne monopolisent plus les vitrines d'exposition. Le rayonnement de masse de l'œuvre littéraire peut ainsi être présenté au travers de l'exposition par la présentation d'innombrables éditions dont a fait l'objet le texte depuis sa première parution: aussi n'estil pas rare de voir exposés aujourd'hui des exemplaires d'ouvrages contemporains ${ }^{34}$, d'éditions bon marché, voire des livres au format de poche ${ }^{35}$, familiers d'une grande majorité des visiteurs ${ }^{36}$. Des livres de format et de genre jugés jusque-là peu conformes au canon du musée et de la prétendue «bonne culture» font également leur entrée au répertoire des expositions: bande-dessinée, mangas, sciencefiction ${ }^{37}$... Témoins de la longue et glorieuse postérité d'une œuvre et d'un auteur, ces ouvrages s'adressent aussi au public de façon plus directe et parlante: le visiteur-lecteur se reconnait dans l'objet présenté et voit dans sa contemporanéité une preuve supplémentaire de l'actualité et de la légitimité du sujet traité par l'exposition.

\section{Qu'exposer du livre, qu'exposer de la littérature dans le livre?}

Les expositions portent la marque de l'identité de l'institution patrimoniale et culturelle dans lesquelles elles ont lieu. Les dispositifs muséaux, d'ordre discursif et scénographique notamment, orientent en cela la réception du propos auprès des visiteurs, à la manière du discours de l'éditeur auprès des lecteurs. Ainsi, la dimension commémorative et la charge symbolique et événementielle de la majorité des expositions littéraires étudiées se cristallisent autour du contenu des livres et en particulier autour de "pages choisies" de la littérature (ainsi de l'exposition consacrée à Hugo par exemple). Plus facilement identifiables par le visiteur idéal (supposé cultivé), ces textes font figure de référence pour la communauté des lecteurs (l'exposition récente de la Fondation Martin Bodmer interrogeait

34 Gallimard. Un siècle d'édition (IgII-20II), Paris, BnF, 22 mars - 3 juillet 2011.

35 Le Musée des civilisations de l'Europe et de la Méditerranée présentait une section littéraire autour d'exemplaires contemporains d'ouvrages critiques et littéraires dans l'exposition Noir et bleu. Un rêve méditerranéen, Marseille, Musée des civilisations de l'Europe et de la Méditerranée, 7 juin 2013 - 6 janvier 2014.

36 À ce sujet, la démarche de la Fondation Martin Bodmer est exemplaire dans l'exposition Le lecteur à l'ouvre, Genève, Fondation Martin Bodmer, 27 avril - 25 août 2013: le lecteur était présenté comme co-réalisateur du sens et de la réception de l'œuvre.

37 Science et fiction, aventures croisées, exposition réalisée avec la $\mathrm{BnF}$, Paris, La cité des sciences et de l'industrie, 2I octobre $2010-3$ juillet 201 . 
cet aspect là encore $)^{38}$. Mais dans la plupart des cas, le mode de présentation sur deux pages implique une certaine hiérarchie, du moins une préférence entre les pratiques et les genres littéraires. La présentation typographique des textes de poésie sur une pleine page permet par exemple de souligner la valeur exemplaire du chef-d'œuvre et du texte canonique. Les romans à succès, illustrés avec brio de préférence, constituent également des éléments intéressants. De même des débuts de chapitres et de paragraphes dont les lettrines et les titres attirent le regard, ou des frontispices consacrant sur une même page les noms de l'auteur et de l'éditeur et le titre de l'œuvre. Autobiographie et essai sont souvent mis en valeur parallèlement quand il y a lieu, au détour de la présentation biographique de l'écrivain. Quant au théâtre $^{39}$ et à la critique littéraire, des documents iconographiques (dessins de costumes, esquisses de décors, peintures) et des captations de performance soulignent la portée scénique et visuelle des deux genres et appuient bien souvent la valorisation du texte. En bref, les pages choisies présentent dans la plupart des cas un intérêt visuel remarquable à partir duquel la scénographie prend forme.

\section{La réification de la littérature à l'œuvre dans l'exposition littéraire}

Dès lors, l'exposition littéraire remet en perspective la place du livre dans le champ littéraire en procédant non pas tant à une sorte d'esthétisation de la littérature qu'à sa réification. En ce sens, le retour sur le devant de la scène de l'auteur depuis le Nouveau Roman et l'affirmation de la Nouvelle Critique, conjugué à un regain d'intérêt pour la biographie et les «lieux de mémoire», assure aux objets évoquant l'Auteur, son milieu et son activité une place de choix dans l'exposition littéraire. Le développement de l'audiovisuel (adaptations théâtrales et cinématographiques, documentaires télévisuels), d'évènements littéraires divers (lectures, conférences) et surtout d'autres supports de lecture (tablettes, livres audio) ont nourri, de leur

$38 \quad$ Néanmoins, ces présupposés entrent peut-être de moins en moins en résonance avec la réalité des publics d'aujourd'hui dont les institutions connaissent de plus en plus précisément le profil. Les "visiteurs réels" sont de plus en plus les destinataires des expositions.

39 Aurélie Rezzouk a travaillé sur le sujet dans sa thèse de doctorat «Exposer le théâtre. De la scène à la vitrine», Paris, Université de Paris-Sorbonne, École doctorale de littératures françaises et comparées, 20I3, 357 p. En cours de publication. 
côté, la diversification des supports sur lesquels peuvent s'appuyer les commissaires d'expositions. Aussi la réification à laquelle procède la muséalisation assure-t-elle à la littérature une dimension spectaculaire et tangible de façon élargie.

Au terme de ce premier temps de réflexion, l'exposition littéraire semble puiser sa source auprès de deux éléments importants qui distinguent les livres rares exposés des livres de consommation courante: l'industrialisation du mode de production et de diffusion du livre d'une part, et le culte du grand écrivain d'autre part. L'ouverture des lieux de conservation de collections littéraires (fonds d'écrivains, bibliothèques) à une action culturelle médiatique de nature patrimoniale en vue d'un public élargi au-delà des seuls lecteurs depuis les années I980 n'a pas fondamentalement changé la donne: le livre demeure au cœur de l'exposition, comme au cœur des collections patrimoniales dans la plupart des cas. Certes, on note l'affirmation de la place dévolue aux manuscrits et aux supports multimédia et interactifs (bornes vidéo, commentaires écrits ou oraux optionnels sur tablettes). Quand les premiers dévoilent les arcanes du processus de création, les seconds incitent le visiteur à s'approprier l'exposition. Les initiatives les plus singulières voient néanmoins le jour dans des lieux peu familiers de la littérature: les musées.

\section{Exposer la littérature «hors du livre»: la mise à l'honneur du texte et de la lecture}

La sortie hors du livre, pleinement revendiquée et assumée, ou ponctuelle selon les expositions, entre en résonance avec la montée en puissance des pratiques contemporaines de lecture s'appuyant sur un support différent du livre papier, hérité du codex. Elle traduit ainsi la volonté de renouveler la voie traditionnelle d'accès à la littérature par le livre papier en redonnant un second souffle à la lecture des textes littéraires perçus comme la citadelle imprenable d'une certaine élite culturelle ${ }^{40}$ et comme un modèle d'autorité dépassé et peu séduisant d'un point de vue muséographique. Cette sortie hors de

40 Pierre Bourdieu et Jean-Claude Passeron, Les Héritiers, Paris, Minuit, I964, I89 p. Pour une synthèse de l'étude des pratiques liées aux livres en France, voir Olivier Donnat, Les pratiques culturelles des Français à l'ère numérique. Éléments de synthèse 1997-2008, Culture études, $\mathrm{n}^{\mathrm{0}}$ 5, 2009, p. 6, http://www. pratiquesculturelles.culture.gouv.fr/o8synthese.php. 
la «culture livre» s'accompagne dès lors de l'entrée de la littérature dans les musées.

\section{Expositions littéraires en bibliothèque et au musée : une pluralité d'approches}

L'approche des musées s'émancipe globalement des deux piliers jusque-là incontestés que sont le livre et l'écrivain: collections et champs d'intervention muséaux different en effet de ceux des bibliothèques. Si le livre continue d'y jouer un rôle important, d'autres objets, supports et voies de médiation prennent aujourd'hui plus d'importance. La ligne de démarcation entre les institutions bibliothécaires et muséales n'est pourtant pas si nette: le développement des expositions littéraires dans les musées souligne l'intérêt des musées pour le monde des bibliothèques. Inversement, les bibliothèques dotées d'une politique d'action culturelle empruntent de plus en plus en la matière aux musées certains de leurs atouts, parmi lesquels l'exposition scénographiée fait figure de proue. La sortie du livre s'explique ainsi par l'interpénétration ${ }^{4 \mathrm{I}}$ des politiques de valorisation des collections patrimoniales, auxquelles s'ajoutent des impératifs communs de communication et d'attraction des publics de plus en plus nombreux et variés.

\section{Présence du livre et image du texte}

Le statut de la littérature comme objet d'exposition n'en demeure pas moins problématique dans tous les cas. De fait, ni le livre, ni le texte n'y sont vraiment exposés pour eux-mêmes et dans leur intégrité, à la différence d'une peinture ou d'une sculpture.

Dans cette perspective, le livre est présenté fermé pour mettre en avant les qualités plastiques de sa couverture ou pour mettre l'accent sur sa matérialité: son contenu et les pages sont alors neutralisés, en même temps que l'expérience sensorielle qui s'y rapporte habituellement (odeur et grain du papier...). On notera que la mise en ligne sur internet des expositions in situ (par exemple des

4I Le cas de la Fondation Bodmer, bibliothèque-musée, est exemplaire de ce point de vue. 
"Brouillons d'écrivains») neutralise en cela la dimension reliquaire et tangible de l'objet fétiche.

À l'inverse, si le livre est ouvert sur un double feuillet, son contenu et ses pages sont figés à un endroit donné. Le texte est immobilisé, comme un film par un arrêt sur image: la présentation du texte, composé d'un ensemble décomposable de signes, d'une unité synthétique de mots sur un support, tel un dessin, donne apparemment à voir une image de texte, plutôt qu'un texte. Bien plus, les conditions d'exposition ne favorisent pas toujours l'appréhension du texte par la lecture, quand le cartel de description de la pièce exposée suffit bien souvent à identifier l'objet illustrant un point donné. Ainsi, le texte ne peut pas toujours faire l'objet d'une lecture analytique malgré l'effort muséographique de mise en valeur de la page (jeu de lumière, isolement dans une vitrine conçue ad hoc...) : le visiteur en prend généralement connaissance en diagonale ou bien la lecture attentive est reportée à l'après-exposition.

\section{L'exposition virtuelle : un cas extrême d'exposition hors du livre?}

Encore à ses débuts, le cas très intéressant des expositions virtuelles présente un modèle singulier de présentation et d'appréhension du texte et du livre. On évoquera ici brièvement quelques pistes de réflexion portant sur des expositions développées par la $\mathrm{BnF}$ en complément de son programme d'expositions physiques, dont les dispositifs multimédia ont inspiré la démarche. La présentation du livre y a tout d'abord ceci de particulier qu'elle y est dématérialisée. Mais ce que l'exposition virtuelle met surtout en valeur de manière réellement frappante par rapport à l'exposition in situ, ce sont de nouvelles façons d'approcher la relation particulière du texte et du signe à la lecture. La présentation de pages du livre induit en effet un rapport singulier au texte qui établit une dialectique particulièrement forte entre le tout et ses parties ${ }^{42}$. De fait, la présentation d'un extrait et d'un éventuel hypertexte induit une séquentialisation du contenu qui trouble la continuité de la lecture et l'appréhension globale du texte, en amont et en aval de l'extrait choisi. En cela certes, rien ne differe de l'exposition réelle. Les capacités technologiques de

42 Anthony Grafton, La page de l'Antiquité à l'ère numérique. Histoire, usages, esthétiques, Paris, Hazan, 20I2, 263 p. Les expositions virtuelles de la $\mathrm{BnF}$ et du réseau des Bibliothèques municipales de Grenoble constituent de bons exemples de cette pratique. 
l'exposition virtuelle conduisent en revanche à s'interroger sur les deux voies extrêmes de présentation du livre: soit que la captation numérique des pages illisibles du livre ouvert ne réduise le texte à une image de livre et de texte, soit (au contraire) que le texte (entièrement) numérisé s'offre au lecteur non pas sur deux pages fixes mais de manière dynamique, à la manière d'un livre électronique dont on peut faire défiler les pages. En ce sens, l'exposition virtuelle, certes onéreuse sur le plan technologique, constitue un moyen de valoriser des collections numérisées par les bibliothèques sans faire encourir le moindre risque matériel aux objets quoiqu'au détriment de l' "aura" du livre. Elle présente néanmoins l'avantage d'être pérenne et accessible au plus grand nombre de visiteurs-lecteurs disposant d'une connexion au serveur de l'institution hébergeant l'exposition, et ce, indépendamment d'expositions réelles très contraignantes d'un point de vue logistique. À terme, enfin, l'exposition virtuelle est susceptible de réaliser le rêve de tout lecteur de constituer sa bibliothèque virtuelle mais aussi, pourquoi pas, son exposition virtuelle. On peut imaginer sur ces plateformes l'insertion d'un onglet spécial permettant de sélectionner des collections numérisées dans un espace virtuel et une modalité de partage du contenu sur les réseaux sociaux comme c'est le cas dans certaines expositions d'art ou d'histoire ${ }^{43}$.

\section{Études de cas}

La question que pose l'exposition de la littérature au travers du livre tient donc à ce qui est montré de la littérature, à l'idée et aux représentations véhiculées: à cet égard, chaque exposition contribuerait à redéfinir les contours de la littérature à sa façon. Les expositions littéraires dans des musées d'art ou d'histoire participent de cette nouvelle donne perpétuelle.

\section{La littérature s'invite au Louvre}

Parallèlement aux musées consacrés aux techniques et à l'histoire du livre ${ }^{44}$, certains musées d'art mettent en œuvre de façon accélérée

43 De nombreux musées proposent déjà cela : le MOMA à New-York, par exemple.

44 Musée de l'imprimerie à Lyon (http://www.imprimerie.lyon.fr/imprimerie/) et exposition Le livre dans tous ses états, Bourges, Musée des Meilleurs ouvriers de France, 7 juillet 2012 - IO mars 2013, par exemple. 
aujourd'hui une politique d'ouverture à la littérature. Avec l'exposition Livre / Louvre ${ }^{45}$ dont le jeu homophonique justifie pour ainsi dire la démarche, le musée parisien a cherché à redéfinir sa programmation et son champ d'intervention. Alors que le musée invite déjà depuis quelque temps des écrivains et des intellectuels à poser un regard sur les collections du musée, la collaboration voulue pour cette exposition semble aller plus loin encore. L'écrivain Jean-Philippe Toussaint y a présenté récemment des installations dans "un hommage visuel à la littérature ${ }^{46}{ }$. Ce parti-pris pouvait s'expliquer par le caractère volontiers décalé de l'entreprise et le désir de relever un projet muséographique aux allures de défi.

Pourtant, force est de constater que l'écrivain-plasticien ne s'affranchissait pas complètement du livre. L'exposition ne cessait en effet de multiplier les représentations liées à cet objet par sa mise en situation au travers de la thématique de la lecture. Horizon du texte littéraire, le livre restait en réalité omniprésent sous de multiples aspects : radiographie de l'auteur lisant un livre qu'on devinait à sa couverture marbrée, photographies de lecteurs tenant un livre en main, exemplaire de La Salle de bain de Jean-Philippe Toussaint posé dans une cabine de douche jusqu'au clin d'œil au canon littéraire. Par la présentation décalée d'un livre hermétiquement clos, un exemplaire très rare de la huitième édition de la Divine Comédie de Dante ${ }^{47}$, le musée d'art semblait en effet discuter les paradigmes du littéraire: confrontation entre un des "pères" de la littérature occidentale, Dante, et Toussaint, un auteur contemporain des Éditions de Minuit, mise en regard entre l'écriture et la création plastique sur des supports contemporains (néons, radiographie, photographies). L'exposition du livre de Dante, présenté tel un bloc fermé et un trésor ${ }^{48}$, interrogeait plus particulièrement les pratiques de lecture en confrontant un monument littéraire à un dispositif contemporain composé de tablettes numériques, traduction matérielle de la diffusion globalisée que connaît la littérature depuis plusieurs décennies. De la sorte, le

\footnotetext{
45 Exposition Livre/Louvre, Paris, Musée du Louvre, 8 mars - II juin 2012.

46 Livre-louvre.arte.tv.

47 Ouvrage tiré du fonds Rothschild dont la valorisation motivait fondamentalement l'exposition, co-réalisée avec le conservateur du patrimoine (et écrivain) Stéphane Torres.

48 Le mode de présentation muséologique ne revêt pas de normes fixes. Toutefois, il est possible de voir dans l'exposition verticale des pièces de musée une certaine esthétisation à laquelle s'opposerait une présentation horizontale davantage documentaire (cf. note 5 ).
} 
musée semblait se faire l'écho des débats contemporains portant sur la disparition du livre papier. Ultime indice: une programmation visuelle plurilingue (un atout pour le public international du musée!) représentait le rougeoiement de la page numérique. Elle mettait en abîme en quelque sorte la "précarité" du codex comme support historiquement déterminé, condamné à la dégénérescence par le livre numérique dont l'écran simulait l'embrasement avant que la page ne renaisse de ses cendres. Enfin, l'exposition invitait justement (sans le vouloir sans doute) à relativiser la notion de "dématérialisation" utilisée de nos jours pour traduire les pratiques actuelles relatives au livre et à la lecture. La diversité et la profusion des installations mises en place dans l'espace d'exposition (tablettes, tirages photographiques, néons) donnaient en effet à voir une multitude de supports matériels au milieu desquels l'héritier du codex, l'exemplaire de Dante, prenait un relief extraordinaire.

\section{Immersion dans «l'univers de Michel Tremblay»}

Dans les expériences évoquées plus haut, le livre reste plus ou moins présent comme point d'ancrage de l'exposition. Il s'agit bel et bien pour l'institution muséale de situer plus largement l'événement dans ses collections, mais aussi dans un domaine de référence susceptible de mettre à l'honneur un savoir-faire et un champ de compétences définis. Cependant, à mesure que les technologies muséographiques évoluent, la plupart des institutions tentent, on l'a dit, d'utiliser ces nouveautés pour rendre la littérature plus accessible à un grand nombre de lecteurs et sous un jour nouveau. Ainsi, des musées de société s'approprient le terrain comme le Musée de la civilisation à Québec ${ }^{49}$.

Le musée québécois a consacré en 20I2-I3 une exposition monographique à l'écrivain québécois Michel Tremblay ${ }^{50}$ sans avoir

49 L'intérêt pour un exemple nord-américain dans une réflexion menée jusque-là en contexte français nous est dicté par le dialogue riche et complexe qui rapproche la France et le Québec. L’appropriation par le Québec des paradigmes propres à la constitution de la littérature (panthéon national, rapport à l'institution scolaire) permet d'observer l'influence nord-américaine et la singularité du modèle québécois, tout en soulignant, par un effet de contraste, le cas français.

so Exposition L'univers de Michel Tremblay, Québec, Musée de la civilisation, I4 mars 2012 - I8 août 2013 . 
recours au livre (papier ou numérique). L'entretien conduit ${ }^{5 \mathrm{I}}$ auprès d'une des actrices du projet, Lise Bertrand, a souligné la volonté ferme du musée de mettre en avant l'œuvre de Tremblay sans passer par le livre, jugé peu «muséal». Les commissaires craignaient effectivement que le livre ne restreigne l'accès à la dimension orale, populaire et «théâtrale» de l'œuvre de Tremblay. Par là, l'exposition redéfinissait la littérature et le statut de l'écrivain en s'affranchissant de l'autorité du livre imprimé au profit d'une interprétation des textes se fondant sur des performances et sur une scénographie muséale insistant sur un aspect de work in progress. En cela, elle se distinguait des autres expositions étudiées jusqu'alors évoquées par l'importance remarquable qu'elle attribuait à l'oralité (interviews de l'auteur projetées sur écran, vidéos retransmettant des scènes jouées, audioguide), au théâtre (comme genre et dans les effets scénographiques) et à un répertoire littéraire davantage marqué par sa réception populaire. Ce choix faisait entrer un pan entier de la littérature contemporaine jusqu'alors peu habitué à obtenir les faveurs du musée, où les écrivains du panthéon scolaire continuent de focaliser l'attention des grandes institutions nationales, voire locales.

Pour se conformer à l'«esprit» de l'œuvre, le musée avait opté pour un décor immersif mettant en scène visuellement, dans le droit fil de l'œuvre dramatique de Tremblay, des décors évoquant certaines de ses œuvres et une atmosphère montréalaise, ville dans laquelle l'écrivain a passé son enfance et qui infuse son œuvre. Ce faisant, le musée privilégiait une «muséologie de point de vue», conformément à une tendance actuelle, ainsi que le constate Jean Davallon: "les expositions-spectacles [qui] régissent le déplacement des visiteurs par des scénographies fortes (mise en scène spatiale ou théâtrale); ou bien [qui] prennent en charge le visiteur au moyen d'un système d'audio-guidage». Et d'ajouter, qu'elles font «naviguer [les visiteurs] à l'intérieur de la matérialisation d'un espace imaginaire qui sert d'enveloppe à ce qu'il rencontre ${ }^{52}$ ". La distinction qu'opère ici Jean Davallon permet de prendre conscience de la portée essentielle de la réflexion menée sur le rapport qu'entretient la littérature à ses supports. En effet, la littérature est peut-être avant tout un «espace imaginaire» constitué de mots. Cet aspect invite à inscrire la littérature dans la notion de "patrimoine immatériel ", avant même que ne soit interrogée la question des supports sur lesquels s'est fixée cette parole.

\footnotetext{
51 Lise Bertrand, entretien téléphonique, 22 février 2013.

52 Jean Davallon, "Le musée est-il vraiment un média?", Publics et Musées, $\mathrm{n}^{\circ}{ }_{2}$, I992, p. II5.
} 
Par là même, il semble que les conditions de transmission du littéraire dans une culture dominée par l'écrit et par le livre, par le culte des reliques et des témoignages du passé que traduit parfaitement le phénomène de muséalisation, conferent à la littérature une dimension matérielle très importante, au fondement du geste d'exposition et des collections de musée.

Pour en revenir à l'exposition étudiée, le musée québécois cherchait précisément à montrer un univers littéraire (un autre mot pour "espace imaginaire»?), irréductible au livre ${ }^{53}$. À l'entrée de l'exposition, Michel Tremblay nous présentait sa vision du monde dans plusieurs interviews dont le montage dessinait une cohérence d'ensemble sur la base de la sélection de thèmes importants dans son œuvre (rôle de la famille, récit de la vocation et du statut donné à l'écriture selon les genres pratiqués). Les décors se trouvaient ainsi conjugués discrètement à la biographie et à certains lieux de vie transposés dans l'œuvre. Dans l'espace suivant, l'œuvre de Tremblay se déployait au plan visuel et sonore par la projection de séquences captant l'interprétation théâtrale de comédiens sur les murs. Cette section nous entraînait dans le décor de l'œuvre comme dans un livre ouvert, dans lequel nous aurions évolué en trois dimensions aux côtés des personnages-comédiens.

Déconcertante mais très réussie, l'exposition québécoise avait donc le mérite d'offrir une alternative à un type d'exposition littéraire qui repose sur la monstration de livres, et à la vision très biographique des expositions monographiques établies sur le canon traditionnel du texte scolaire. Elle redéfinissait en quelque sorte les contours du répertoire et des conventions littéraires largement reconduites dans les musées.

\section{Conclusion}

Qu'est-ce qu'un livre? Emmanuel Kant répond à cette question dans la seconde partie des Fondements de la métaphysique des moeurs (1785): un livre est un objet produit par un travail de manufacture, mais il s'agit également d'une ouvre, d'un discours. Qu'est-ce qu'exposer la littérature? Qu'exposer d'elle? La complexité soulevée par Kant se

53 L'abondance des supports utilisés (écrans en tous genres notamment) ne doit pas masquer une des limites du terme "dématérialisation", qui peut dès lors paraître étrange. Voir notamment: Yves Jeanneret, $Y$-a-t-il (vraiment) des technologies de l'information?, Villeneuve d'Ascq, Presses Universitaires du Septentrion, 2007, $200 \mathrm{p}$. 
pose en des termes voisins: la littérature est à la fois une œuvre, un ensemble homogène de signes écrits consignés sur un support mais également un «discours». Toutefois, elle n'est pas réductible à l'écrit et encore moins assignable à un support déterminé. Son exposition relève donc de la gageure et prend des formes extrêmement variées. Pourtant, le livre conserve une position privilégiée et constitue un horizon essentiel de la question. L'exposition littéraire contribue à interroger les contours de la littérature dans le rapport qui lie son support historique au "texte» qui en constitue l'alpha et l'oméga. En effet, si le livre occupe une place aussi essentielle dans les expositions littéraires, n'est-ce pas dû à la difficulté qu'il y a à s'émanciper du texte et de l'écrit comme horizon de la littérature et à considérer celleci comme «immatérielle»? Le texte dont l'étymologie et les usages ont fini par confondre la trame du papier avec la trame des mots ... Par conséquent, la présence du livre dans l'exposition est davantage à considérer sur le plan de tout un modèle culturel construit autour du couple livre-littérature que sur le plan d'un simple support.

Enfin, en interrogeant la création littéraire sous un jour inhabituel, décalé ou critique, l'exposition met en question le statut d'écrivain. Son essor, au-delà de la seule bibliothèque, traduit en effet à sa façon l'impérieuse nécessité d'être visible pour tout écrivain cherchant à exister en tant que tel dans notre monde moderne, et particulièrement dans la société contemporaine. La présence de l'auteur sur la scène publique démultiplie les événements au cours desquels il se voit "exposé». L'exposition de l'écrivain, telle que l'envisage le sociologue Bernard Lahire ${ }^{54}$, passe semble-t-il par l'affirmation d'une présence de l'écrivain comme sujet, objet ou commissaire d'exposition. De l'exposition-sanctuaire à l'exposition médiatique, le musée renvoie à l'écrivain un miroir intéressant de sa condition.

\section{Expositions : corpus primaire}

Le livre dans tous ses états, Bourges, Musée des meilleurs ouvriers de France, 7 juillet 2012 - IO mars 2013, http://www.ville-bourges.fr/ site/culture_livre-etats.

54 Bernard Lahire, Ce qu’ils vivent, ce qu'ils écrivent. Mises en scène littéraires $d u$ social et expériences socialisatrices des écrivains, Paris, Éditions des archives contemporaines, 2011,569 p. Les questions abordées ici feront l'objet en juin 2015 d'un numéro spécial de la revue Interférences littéraires / Literaire Interferenties dirigé par M.-C. Régnier. 
Livre/Louvre, Paris, Musée du Louvre, 8 mars - II juin 20I2, http:// livre-louvre.arte.tv/.

L'univers de Michel Tremblay, Québec, Musée de la civilisation à Québec, I4 mars 20I2 - I8 août 20I3, images intéressantes sur le lien: http://www.lapresse.ca/le-soleil/arts-et-spectacles/ expositions/20I203/I3/OI-4505215-michel-tremblay-a-lhonneur-aumusee-de-la-civilisation.php.

\section{Expositions : corpus secondaire}

Le Lecteur à l'aeuvre, Cologny, Fondation Martin Bodmer, 27 avril - 8 septembre 2013, http://fondationbodmer.ch/2012/o5/le-lecteura-l-oeuvre/.

Noir et Bleu. Un rêve méditerranéen, Marseille, Le Musée des civilisations de l'Europe et de la Méditerranée, 7 juin 20I3 - 6 janvier 20I4, http://www.mucem.org/fr/exposition/716.

Les Misérables, un roman inconnu? Paris, Maison Victor Hugo à Paris, IO octobre $2008-\mathrm{I}^{\mathrm{er}}$ février 2009, http://maisonsvictorhugo.paris.fr/ fr/expositions/les-miserables-un-roman-inconnu.

Balzac vu d'ailleurs. Un regard tä̈wanais sur La Comédie humaine, Paris, Maison de Balzac à Paris, I ${ }^{\text {er }}$ mars -26 mai 2013, http:// parismusees.paris.fr/fr/exposition/balzac-vu-dailleurs.

La légende du roi Arthur, Paris, Bibliothèque nationale de France, Site François Mitterrand, Grande Galerie, 20 octobre 2009 - 24 janvier 20IO, http://expositions.bnf.fr/arthur.

Antonin Artaud, Dessins, Paris, Centre Georges Pompidou, ${ }^{\text {er }}$ juillet I987 - II octobre I987, http://www.centrepompidou.fr/cpv/resource/ cn $7 \times 5 \mathrm{RX} / \mathrm{rbXenM}$.

Antonin Artaud, Paris, Bibliothèque nationale de France, Site François Mitterrand, 7 novembre 2006 - 4 février 2007, www.bnf. fr/documents/cp_artaud.pdf.

Pierre-Jean Jouve, Paris, Bibliothèque nationale de France, Site François Mitterrand, 24 septembre 20I3-IO novembre 20I3, http:// www.bnf.fr/fr/evenements_et_culture/anx_expositions/f.pierre_jean_ jouve.html?seance $=\mathrm{I} 22391098585 \mathrm{I}$. 


\section{Expositions virtuelles (à partir d'expositions in situ) :}

L'aventure du livre, Paris, Bibliothèque nationale de France, http:// classes.bnf.fr/livre/.

Choses lues, choses vues, Paris, Bibliothèque nationale de France, site Richelieu, Salle Labrouste, 23 octobre 2009-3I janvier 20IO, http:// expositions.bnf.fr/lecture/.

Victor Hugo, l'homme-océan, Paris, Bibliothèque nationale de France, site François-Mitterrand, 2I mars - 2I juin 2002, http://expositions. bnf.fr/hugo/.

Brouillons d'écrivains, Paris, Bibliothèque nationale de France, site François-Mitterrand, 27 février - 24 juin 200I, http://expositions. bnf.fr/brouillons/.

Gallimard. Un siècle d'édition (I9II-20II), Paris, Bibliothèque nationale de France, Galerie François I ${ }^{\mathrm{er}}, 22$ mars -3 juillet 20II, http:// expositions.bnf.fr/gallimard/.

\section{SUMMARY}

From Renaissance collections to nineteenth-century print exhibitions, the "museumification" of literature calls for a redefinition of the traditional relationship between literature and books. Based on a sample of literary exhibitions in museums, this article examines the issues they bring to the fore on a period that extends from the end of the nineteenth century to today. It adopts a multidisciplinary approach to investigate the theoretical and practical underpinnings of the concomitant museumification of literature and books by observing the tensions that it creates on an institutional scale, both between libraries and museums and within certain literary paradigms (genres, "classics," etc.). This article thus explores the motivations that might explain the seemingly central place of the book in the literary exhibition as opposed to a multitude of objects a priori more spectacular. In this sense, the hyper-materiality of literature and its dissociation from the book (on which the very existence of the literary museum is paradoxically based) is the focus of detailed analysis here. The literary exhibition relies on the contextualization of the literary text and, especially in a time of emphasis on digital text, 
on its place in the history of the book. Museumification today tends to attribute additional value to literature that is on other platforms than paper as a way of adding a spectacular aspect to the exhibition. In this perspective, the contemporary "dematerialization" of the book poses an ultimate question - a question concerning digital literary collections and the use of digital platforms in the added value of literature. By facilitating and broadening the access to heritage, virtual exhibits (one example of the digital platform) offer the double advantage of protecting fragile collections and making their value more durable. They run the risk, however, of neutralizing the "aura" of paper books and the quest for tangible objects, which constitute the main reason for our society's attachment to paper books and the foundation of museums. 
